\title{
THE EFFECT OF PHYTOCOMPOUNDS OF MEDICINAL PLANTS ON CORONAVIRUS (2019-NCOV) INFECTION
}

\author{
Ahlem Zrig1,*
}

Original article submitted October 16, 2020.

The recent outbreak of coronavirus disease 2019 (COVID-19) is a respiratory infection and it can spread from animal to person, person to person through coughing or physical contact. Recent studies revealed that the genome sequence of this pandemic disease is very similar to that of SARS-CoV, thus, there are not yet effective treatments that target the 2019-nCoV virus. Medicinal plants present a potential solution to resolve the drug problem. In fact, the bioactive compounds such as phenols, flavonoids, monoterpenes, and phenylpropanoids, derived from several herbs such as Eucalyptus globulus, Mentha spicata, Nigella sativa, Rosmarinus officinalis, Thymus capitatus and Zingiber officinale could be used to develop formal drugs against several diseases with no or minimal side effects. In this paper, we describe the potential antiviral properties of several medicinal plants against Coronaviridae family viruses such as SARS, MERS, and IBV. Besides, we review various species of medicinal herbs and their derived phytochemical compounds in terms of their immunomodulatory bioactivities and antiviral activity.

Keywords: COVID-19; medicinal plants; phytochemical compounds; antiviral activity.

\section{INTRODUCTION}

Since December 2019, a novel form of virus belonging to Coronaviridae family [1] and the related coronavirus disease (COVID-19), was identified in Wuhan of China according to the World Health Organization (WHO). In the beginning, this virus was transformed from animal to human, thereafter, the Chinese health authorities reported human to human transmission regarding the sharply increase in the number of persons infected. Since then, case counts have been increasing rapidly in china and in several countries in Asia, Europe, North America and Africa. Until now, World is in great depression and it is experiencing the second wave of COVID-19 which has affected majority of the countries with 44,966,876 confirmed cases and about 1,181,721 confirmed deaths as of 29th October 2020. In Tunisia, COVID-19 statistics are 54,278 infections and 1,153 deaths. According to scientist, the number of cases will be higher by next months and this pandemic is continuing all around the world. The new COVID-19 primarily attacks the lower respiratory system [2]. After period between 2 to 14 days, which is described as an incubator period for the virus, many symptoms

\footnotetext{
${ }^{1}$ Research Unit of Biodiversity and Valorization of Bioresources in Arid Zones, Faculty of Sciences of Gabès-City Erriadh, Zrig, Gabes 6072, Tunisia.

* e-mail: ahlem18zrig@yahoo.fr
}

start to show up in the infected person such as cough, fever, myalgia, fatigue, shortness of breath. Moreover; diarrhea, chest pain, confusion, nausea-vomiting, headache, hemoptysis and hyposmia were observed. Hence, pneumonia appears to be the most manifestation of new COVID-19, but some extrapulmonary involvement such as gastrointestinal, cardiac and renal has been reported [3]. Unfortunately, until now there is no specific treatments able to cure from COVID-19 in short time. There are some suggestions to use the chloroquine and hydroxylchloroquine drugs which have been identified as inhibitors of growth for many different viruses such as SARS coronavirus, human coronavirus OC43, enterovirus EV-A71, Zika virus and influenza-A H5N1. Many studies reported that genetic similarities between the new coronavirus (2019-nCoV) and SARS or MERS coronaviruses are high [4]. In addition, to prevent and treat viral infection caused by 2019-nCoV, the development of specified treatments may require months or years, meaning that a more immediate treatment or control mechanisms should be found if possible [5].

Thus, the situation required to discover new preventive and therapeutic agents as soon as possible. In this sense, medicinal plants have attracted significant attention because they include natural and bioactive compound that could be used as anti-coronavirus (2019-nCoV) by developing formal drugs. Therefore, the development of drug candidates from various traditional or alternative and complementary medi- 
cines is receiving increasing worldwide attention [6]. With the large number of phytochemicals discovered in many aromatic herbs, some compound may be extracted and used as a primary compound to produce a synthetic antiviral drug. Many countries such as Tunisia, is unable to produce their own drug since they could not acquire the primary compound to their laboratory. In this case, it will be necessary and emergency to explore the antiviral substances derived from medicinal plants not only to produce a new agent anti-coronavirus but also to resolve the deficiency in primary materiel to the generic drugs such as the chloroquine and hydroxychloroquine [7]. Few investigations of antiviral activity of many plants were done compared to their anti-microbial proprieties. Lucky, serval researches demonstrated that the activity of antiviral substances derived from plants was positive in vitro and in vivo [8]. However, same plants can have different antiviral activity against RNA or DNA viruses, either enveloped or non-enveloped, and even against different types or strains of viruses $[9,10]$. The effectiveness of herbal treatment to control contagious disease was demonstrated in severe acute respiratory syndrome (SARS) outbreak $[11,12]$ and several virus belongs to Coronaviridae family. In this review, we focus on recent advances in the research and development of phytocompounds derived from many Medicinal plants and tested in many viruses belongs to coronavirus to find the most effective natural compounds which can be used as anti-coronavirus (2019-nCoV) in short time.

\section{METHODOLOGY}

Our methodology is based on reviewing recent papers devoted to the effect of bioactive compounds of medicinal plants on coronavirus. The scientific literature mainly focusing on plant natural products with therapeutic efficacies against experimental models of coronavirus, were identified and tabulated. In the first step we conducted a literature search for medicinal plants which used to cure from respiratory syndrome coronavirus such as SARS and virus having a genetic similarity to COVID-19. Next, we identified the results of the in vitro evaluations and recognized the natural compounds and their antiviral activity. In the second part, the most compounds derived from medicinal plants were selected to understand how these compounds may inhibit the growth of virus in cell. Finally, tested natural compounds were cross-checked for listing in the Tunisian medicinal plants and having a highest antiviral activity. The selected compounds were not tested against the new coronavirus until now.

\section{IN VITRO EVALUATION OF THE ANTIVIRAL ACTIVITY OF MEDICINAL PLANTS AGAINST COVID-19}

The coronavirus encodes more than one dozen proteins (proteases), some of which are essential to viral entry and replication. Therefore, the enzymatic activity of protease (viral 3-chymotrypsin-like cysteine protease (3CLpro) is essential for the viral life cycle, and it is an attractive target for the development of antiviral drugs directed against coronavirus infections. Khaerunnisa, et al. [13] successfully crystallized the main protease (Mpro)/chymotrypsin-like protease (3CLpro) from COVID-19, which has been structured and repositioned in the Protein Data Bank (PDB) and is accessible by the public. This protease represents a potential target for the inhibition of $\mathrm{CoV}$ replication [14]. The experimental strategy was to investigate the phytocompounds effects on this enzyme, thereafter they inhibit the growth of virus in cell [15]. The screening of a series of small molecules from natural compounds confirmed that those molecules inhibit directly the protein. For example, Zhang, et al. [16] found 13 natural compounds derived from traditional chines medicinal plants having potential anti-2019nCoV activity. The anti-coronavirus effects of the natural compounds screened by these authors have been mainly confirmed in vitro by direct loading onto cultured cells, thus it does not guarantee their effectiveness in vivo. It was also reported [16] on the success of docking screening which was due to high genetic similarity between the new coronavirus and SARS or MERS viruses.

In order to discover new compounds that may serve as potential anti-COVID-19 drugs, Tahir, et al. [1] identified nine new non-toxic druggable natural compounds predicted to bind with the receptor binding site and catalytic dyad (Cys-145 and His-41) of SARS-CoV-2 3CLpro. These selected potential anti-viral phytochemicals may inhibit SARS-CoV-2 3CLpro activity and hence virus replication. Moreover, in view of a high similarity to COVID-19, theses natural compounds could be used as anti-COVID-19 drugs. In the same sense, 16 medicinal plants extracts were evaluated for their cytotoxicity, and seven extracts from D. canadense, $M$. piperita, $M$. officinalis, $O$. vulgaris, $T$. vulgaris, $H$. officinalis, and $S$. officinalis possessed significant anti-IBV activity against coronaviruses belonging to the Coronaviridae family [17]. Furthermore, the same study proved that $M$. piperita, $D$. canadense and T. vulgaris extracts exhibited strongest viral replication inhibition and completely stopped IBV production. Moreover, hydroalcoholic and aqueous extracts of M. piperita and T. vulgaris was shown to exert significant anti-HSV-1 and anti-HSV-2 activities in vitro [17].

Many studies reported that all extract belonging to Lamiaceae family showed antiviral activity. In the search for a coronavirus specific therapy, 1000 traditional Chinese medicinal herbs were screened and 10 active compounds were tested clinically to inhibit the coronavirus [18]. A similar 
study [19] demonstrated that Cimicifuga rhizoma, Meliae cortex, Coptidis rhizoma, Phellodendron cortex and Sophora subprostrata radix exhibited anti-MHV activity suggesting that they contain candidate compounds for anti-coronavirus drugs. The most effective anti-viral phytochemicals selected to inhibit the coronavirus disease were flavonoids or essentials oils.

\section{ACTIVE COMPOUNDS OF MEDICINAL PLANTS}

Usually, the first step in investigating the biological activity (including antiviral) of complex natural products is to determine the most abundant compounds in any type of extract. Several cited works used this approach and selected some compounds for further investigation of their activity against viruses.

\subsection{Antiviral Activity of Phenolic Compounds and Flavonoids}

Flavonoids are natural product derived from major medicinal plants. These phenolic compounds are ubiquinone with pharmacological potency. Flavonoids act as potent antioxidant and are known to display antiviral activities against HSV, dengue virus, influenza virus [20] and SARS-CoV [21]. Many works demonstrated their high inhibition activity in Severe Acute Respiratory Syndrome (SARS) coronavirus and the antiviral properties of some compounds of flavonoids especially against clinically important enveloped viruses belonging to the coronavirus group. It was suggested [22] that the anti-coronavirus activity of some flavonoids (Herbacetin, rhoifolin and pectolinarin) was due to the inhibition of 3C-like protease (3CLpro). Some flavonoid compounds such as quercetin, quercetin derivatives, catechin, epicatechin, epicatechin gallate and epigallocatechin gallate inhibit SARS-3CLpro expressed in Escherichia coli [23]. Besides, it was reported [24] that bioflavonoids from Torreya nucifera also brought inhibition effect on SARS-CoV/3CL and it was demonstrated that amentoflavone isolated from $T$. nucidera was an effective inhibitor of SARS-CoV 3CLpro and was even more effective than the corresponding flavones (apigenin and luteolin) and biflavonoid derivatives containing various numbers of methoxy groups. The IC50 value of this inhibitor (although higher than those of peptide-derived 3 CLpro inhibitors) was nonetheless in the low micromolar range. It was suggested that this compound could be a good candidate for development as a natural therapeutic drug against SARS-CoV infection and therefore against the COVID-19. This compound is the constituent of a number of plants including Ginkgo biloba, Chamaecyparis obtusa (hinoki), and Hypericum perforatum [25]. Another investigation [26] indicated that, among flavonoids compounds, quercetin, luteolin-7-glucoside, demethoxycurcumin, naringenin, apigenin-7-glucoside, oleuropein, curcumin, catechin, and epicatechin-gallate were the most recommended compounds found in medicinal plants possessing the best potential as in- hibitors against COVID-19. These secondary metabolites were extracted from many species such as Spinacia oleracea, Brassica oleracea, Anethum graveolens, Brassica rapa, Sauropus androgynus, Anethum graveolens, Foeniculum vulgarea, Allium cepa, Oregano vulgare, Capsicum annum, Olea europaea, Averrhoa belimbi, Capsicum annum and Allium fistulosum. Jo, et al. [27] reported that herbacetin, isobavachalcone, quercetin 3- $\beta$-d-glucoside, and helichrysetin were found capable of blocking the enzymatic activity of MERS-CoV/3CLpro.

Other ubiquinone substances were also examined in several plants. Chalcones isolated from A. keiskei were found to act as inhibitors against the SARS-CoV proteases [28]. Investigations of the antiviral activity of natural products reported that isoflavones extracted from Psorothamnus arborescens exhibited the highest binding affinity and formed strong hydrogen bonds with the catalytic dyad residues which disrupt important hydrogen bonds and alter the receptor binding site, thereby affecting its ability to bind with the SARS-CoV inhibitor, and other phenolic compounds such as two abietane-type diterpenoids, carnosol and carnosic acid, have been found in high amount in Rosemary extracts (Rosmarinus officinalis), a frequently used traditional herbal remedy [29]. In addition, antiviral activity of tannins was demonstrated by inhibiting the viral reverse transcriptase. It was reported [30] that tannins and proanthocyanidins inhibited the viral DNA and RNA replication and the flavonoids blocked the viral RNA synthesis. According to all researches presented here, most phytochemicals act as antiviral via two different mechanisms to inhibit the effect of COVID-19. It appeared that the natural compounds exhibited their effects on viral particles (i) either prior to attachment to the host cell or (ii) after the virus enters the host cell. Indeed, flavonoids were reported to exhibit antiviral effects via inhibiting the RNA synthesis of viruses. While the polyphenols acted principally by binding to the protein coat and thus arrested absorption of the virus; they were also reported to inhibit viral replication enzymes (such as RT for HIV and RNA polymerase for influenza virus).

\subsection{Antiviral Activity of Essential Oils}

Essential oils are complex mixtures of compounds with low molecular weights, such as monoterpene hydrocarbons, sesquiterpene hydrocarbons, and their corresponding oxidized products (e.g., alcohols, aldehydes, and ketones); homologues of phenylpropanoids; and small amounts of diterpenoids. The active components of essential oils might consist of lipophilic carbohydrates that interact with the lipid membrane. Much evidence has been reported about antiviral potential of various essential oils and their constitutions on several genera of viruses. Virucidal activity of essential oils, which are lipophilic by nature, is probably due to disruption of the viral membrane or interference with viral envelope proteins involved in host cell attachment. Nevertheless, the inactivation of vital cycle of the virus by the essential oils is 
time dependent and that infectious virus remaining after treatment are still sensitive to the essential oils making selection of resistant mutants unlikely [31]. Another study was conducted in Salvia officinalis L., Laurus nobilis, Thuja orientalis, Juniperus oxycedrus, Pistacia palaestina Boiss. and Cupressus sempervirens, and the results demonstrated that the major constituent of essential oils extracted from each species directly affected SARS-COV replication in vitro [11]. This work reported that Laurus nobilis berries oil exhibited inhibitor effect against SARS-CoV by their major essential oil including $b$-ocimene $(21.83 \%), 1,8$-cineole (9.43\%), $a$-pinene (3.67\%), and $b$-pinene (2.14\%). Furthermore, certain activity against SARS-CoV was found for $T$. orientalis and $J$. oxycedrus [11]. In some investigations, sesquiterpenes and sesquiterpene-rich essential oils extracted from the leaves of Pogostemon cablin Benth. showed potent in vitro antiviral activity against influenza virus $\mathrm{A} / \mathrm{PR} / 8 / 34$ (H1N1) [32].

Among the terpenoid compounds, terpinen-4-ol, terpinolene, and á-terpineol, show inhibitory effect on influenza A/PR/8PR virus subtype H1N1. Also, it was indicated that some sesquiterpenes such as â-sesquiphellandrene, and tetrahydronaphthalenol, showed potent antirhinoviral activity in a plaque reduction test [33]. Moreover, the main components of Zingiber officinale oil, which are sesquiterpenes (zingiberene, bisabolene, sesquiphellandrene, and curcumen), Thymus vulgaris oil (thymol and carvacrol), and hyssop oil consist mainly of monoterpenes (e.g., 1-pinocamphone, isopinocamphone, pinocarvone, and -pinene), and sandalwood oil composed of sesquiterpene alcohols (e.g., santalol, bergamotol, and santalene) affected the virus before adsorption to or entry into host cells and disrupt the viral envelope [34]. Additionally, these studies have demonstrated the effect of a natural based product consisting of a synergistic blend of botanical oleoresins and essential oils on IBV. The effect appears to be more pronounced on cell free virus indicating that the activity is likely virucidal, which is important because it may also be effective against other enveloped respiratory viruses like COVID19. In addition, since virucidal compounds work by inactivating viruses, rather than targeting specific virus genes or proteins, it reduces the likelihood that resistance will develop from mutations during virus replication [35].

\section{ANTIVIRAL ACTIVITY OF SOME TUNISIAN MEDICINAL PLANTS}

Use of herbal medicines was widely practiced in Tunisia. Few research evaluated the antiviral activity in vitro of some Tunisian medicinal plants. Studies were conducted on fifteen species and the results corroborated those medicinal plants from Tunisia can be a rich source of potential antiviral agents, therefore, their phytocompounds can be used as drugs for COVID-19 treatment. The extract of Plantago major L. (Plantaginaceae) exhibited antiviral activity mediated by their phenolic compounds [36]. Some Hypericum species belonging to Hypericaceae family were used in Tunisian traditional medicine as an antiseptic and for wound healing and it was found that its antiviral activity could be attributed to hypericin, pseudohypericin and some other compounds. In addition, the acetonic and methanolic extracts of $F$. pulverulenta aerial parts inhibited virus (HSV-1) propagation. Another Ericaceae species used in traditional medicine was Erica multiflora, a promising plant with antiviral activity. In Tunisian traditional medicine it was used as urinary antiseptic, astringent, tranquillizer and for treating prostate cancer. Ben Sassi, et al. [36] observed high antiviral activity of Zygophyllum album L. (Zygophyllaceae) and suggested that it was due to the possible presence of triterpenoid saponin. In Tunisian popular medicine, Z. album was used for wound healing and as soap for face and body. Besides, $Z$. album contains tannins which have been reported to exhibit an antiviral activity. Many medicinal plants were used for their higher antioxidant activity. Therefore, the use of antioxidants can be of great value in preventing the inception or the progression of the disease by elevating the level of $\mathrm{O} 2$ before replication in the bronchial epithelial cells occurs. In this sense, it was demonstrated [37] that Marrubium deserti de Noé exhibited significant antiviral activity, and Marrubium deserti de Noé was used in traditional medicine, for example as a remedy for asthma and diabetes, and also found to have antibacterial properties. It is of particular interest in Tunisia and this is why we continued research on this plant. In the first study on antiviral activities of Teucrium pseudochamaepitys, Hamman, et al. [38] reported that the bioactivity evaluation of the essential oils composition demonstrated a more significant antioxidant and antiviral activities in this species. T. pseudochamaepitys belonging to the Lamiaceae family is growing spontaneously in Tunisia and is rich in stronglybioactive phenolic compounds and are used in folkloric medicine, in the food industry and in pharmacies for their antimicrobial, antinociceptive, antioxidant, hypolipidemic, anti-inflammatory and hypoglycemic properties. Further phytochemical investigations of T. pseudochamaepitys crude and volatile extracts to isolate the biologically-active compounds will be of great importance. Salah-fatnassi, et al. [39] investigated the antiviral activity of Thymus capitatus and they reported that the essential oils extracted from this species exhibit a significant inhibition of virus at the adsorption as well as after penetration onto host cell. The genus Thymus is represented in Tunisia by five species, including T. capitatus $(\mathrm{L}$.) whose uses in traditional Tunisian medicine are quite numerous [39].

\section{CONCLUSION}

According to all research on bioactive compounds derived from many medicinal plants, we suggest that the same phytocompounds derived from medicinal plants tested on SARS or IBV can be used for COVID-19 treatment, since 
they are high genetic similarity between the coronavirus. In addition, many medicinal plants were used for in vitro analysis of their bioactive compounds to study their antiviral activity. These plants were found in many and different countries in the world. These countries should develop a scientific program devoted toward modern drug discovery from phytochemicals compounds of plants extracts.

\section{FUNDING}

This research did not receive any specific grant from funding agencies in the public, commercial, or not-for-profit sectors.

\section{CONFLICT OF INTEREST}

The authors declares that he has no conflictstoffinterest.

\section{REFERENCES}

1. M. Tahir, S. M. Alqahtani, M. A. Alamri, et al., J. Pharm. Anal., 10(4), 313 - 319 (2020).

2. K. Ng, B. H. Poon, T. H. Kiat Puar, et al., Ann. Intern. Med., 172(11), 766 - 767 (2020).

3. J. Gu, B. Han, J. Wang, et al., Gastroenterology, 158(6), $1518-1519(2020)$

4. P. Zhou, X. Yang, X. Wang, et al., Nature, 579, 270-273 (2020).

5. L. Zhang and Y. Liu, J. Med Virol., 92(5), 479 - 490 (2020).

6. K. Aravindaram and N. S. Yang, Planta Med., 14(3), $241-246$ (2010).

7. A. K. Singh, A. Singh, A. Shaikh, et al, Diabetes Metab. Syndr. Clin. Res. Rev., 14(3), 241 - 246 (2020)

8. R. Gupta, A. Ghosh, A. Kumar, et al., Diabetes Metab. Syndr. Clin. Res. Rev., 14, $211-212$ (2020).

9. M. F. Visintini Jaime, F. Redko, L. V. Muschietti, et al., Virol. J., 10, 245 (2013).

10. A. Schuhmacher, J. Reichling, P. Schnitzler, et al., Phytomedicine, 10(6 - 7), 504-510(2003).

11. R. Loizzo, A. M. Saab, R. Tundis, et al., Chem Biodivers., 5(3), $461-70.5$ (2008)

12. Z. Chen, and T. Nakamura, Phytother. Res., 18(7), $592-594$ (2004)

13. S. Khaerunnisa, H. Kurniawan, R. Awaluddin, et al., Preprints, 210, 1 - 12 (2020).

14. L. Shen, J. Niu, C. Wang, et al., J. Virol., 93(12), 23 - 19 (2019).
15. C. Wen, H. Chen, N. Yang, et al., Adv Bot Res. 62, $197-272$ (2012).

16. D. Zhang, K. Wu, X. Zhang, et al., J. Integr. Med., 18, 152-158 (2020).

17. R. Lelešius, A. Karpovaite, R. Mickiene, et al., BMC Vet. Res.. 15, 178 (2019).

18. C. Y. Wu, J. T. Jan, S. H. Ma, et al., Proc. Natl. Acad. Sci., 101(27), 10012 - 10017 (2004).

19. H. Kim, H. Shin, H. Park, et al., J. Clin. Virol., 41(2), $122-841$ 122-128 (2008).

20. A. Tapas, D. Sakarkar, R. Kakde, et al., Trop. J. Pharm. Res., 7(3), 1089 - 1099 (2008).

21. S. Jo, S. Kim, D. H. Shin, et al., J. Enzyme Inhib. Med. Chem., 35, $145-151$ (2020).

22. S. Jo, H. Kim, S. Kim, et al., Chem. Biol. Drug Des., 94(6), $2023-2030$ (2019).

23. T. Thanh, H. Nguyen, H. W. H. Kang, et al., Biotechnol. Lett., 34(5), 831 - 838 (2012).

24. Y. B. Ryu, H. J. Jeong, J. H. Kim, et al., Bioorg. Med. Chem., 15, 18(22), 7940 - 7947 (2010).

25. S. Bais, N. Abrol, et al., Curr. Res. Neurosci., 6, 16 - 22 (2016).

26. S. Khaerunnisa, H. Kurniawan, R. Awaluddin, et al., Preprints, $1-14$ (2020).

27. S. Jo, H. Kim, S. Kim, et al., Chem. Biol. Drug Des., $1-14$ (2019).

28. J. Y. Park, J. A. Ko, D. W. Kim, et al., J. Enzym. Inhib. Med. Chem., 31(1), 23 - 30 (2016).

29. H. B. Shin, M. S. Choi, B. Ryu, et al., Virol. J., 8, 10-303 (2013).

30. S. A. A. Jassim, M. A. Naji, et al. J. Appl. Microbiol., 95, 412 - 427 (2003).

31. P. Schnitzler, C. Koch, et al., Antimicrob. Agents Chemother., 51(5), 1859 (2007).

32. H. Kiyohara, C. Ichino, Y. Kawamura, et al., J. Nat. Med., 66(1), $55-56$ (2012).

33. A. Pasdaran, A. Pasdaran, D. Sheikhi, et al., Microbiol. Respirat. System Infect., 237 - 261 (2016).

34. P. Schnitzler, C. Koch, J. Reichling, et al., Antimicrob. Agents Chemother., 51(5), 1859 - 62 (2007).

35. M. W. Jackwood, R. Rosenbloom, M. Petteruti, D. A. Hilt, et al., Virus Res., 149, 86 - 94 (2010).

36. A. Ben Sassi, N. Bourgougnon, M. Aouni, et al., Nat. Prod. Res., 22(1), 53 - 65 (2008).

37. H. Edziri, M. Mastouri, M. Aouni, et al., South Afr. J. Bot., 80, $104-109$ (2012).

38. S. Hammami, H. Jmii, R. El Mokni, et al., Molecules, 20(11), 20426 - 20433 (2015).

39. K. B. H. Salah-fatnassi, A. Slim-bannour, F. Harzallah, et al., Acta Bot. Gallica, 157(3), 433 - 444 (2010) 